\title{
STRUCTURAL AND EVOLUTIONARY FEATURES OF FLARE- PRODUCING ACTIVE REGIONS DURING 1988-1990
}

\author{
A. A. GOLOVKO \\ Institute of Solar-Terrestrial Physics (ISTP AT RAS SD), \\ Irkutsk - 33, P.O.Box 4026, Russia 664033
}

During the period of high solar activity (1988-1990) the Sayan and Baikal Observatories of the ISTP carried out observations of the chromosphere in the $\mathrm{H}$-alpha line, the photosphere and of sunspot magnetic fields; at the Siberian Solar Radio Telescope, solar radio emission observations at $5.2 \mathrm{~cm}$ wavelength were made. Using those observations a study is made of the evolution of "centers of magnetic activity" (Bumba, 1986) in nine active regions with different flare productivity, for which the most complete observations were available. In three cases (NOAA Nos. 5229, 5643 and 5669) they were the targets of the cooperative international Max'91 program.

The active regions under consideration were all characterized by the same evolutionary feature, namely newly emerging flux regions (EFR) at "centers of magnetic activity" of well-developed active regions. Such a characteristic is typical of flare-productive active regions (Rust, 1973). Photospheric and chromospheric data were used to determine the EFR boundaries on the photosphere, and estimates were made of the value of emerging magnetic flux $F$ (of like polarity) for each day of the observation.

Table I gives the EFR characteristics and the data on the number of flares in the ARs studied. The AR No. 5629 (No. 5669 in the next rotation) was the most flare-productive region, and the AR No. 5796 was the least productive region. Comparison of the character of evolution of the active regions resulted in dividing them into two categories. In the first of them (they include ARs Nos. 5409, 5793 and 6368) the period of increased flare activity covers several days and correlates with the evolution of one given emerging flux region that interacts with surrounding parts of the AR ("concentrated magnetic activity"). In the second category of ARs, which includes all the other cases, flares occur almost continuously during the solar disc passage of the region and accompany processes of multiple occurrence of emerging flux regions in different parts of an extended AR ("broken magnetic activity"). In this respect, the most characteristic is the AR No. 5669, in which at least 5 EFRs were identified. 
We have made a combined analysis of the optical data and the one-dimensional solar scans at $5.2 \mathrm{~cm}$ wavelength, obtained with the Siberian Solar Radio Telescope (Smolkov et al., 1990) and kindly made available to us by Dr.B.I.Lubyshev. The analysis has shown that emerging magnetic fluxes at "centers of magnetic activity" correlate with the appearance and evolution of a peculiar radio emission source "between sunspots" as described by Akhmedov et al. (1986). The evolution curves for the time dependence of the value of emerging magnetic flux $F(t)$ and the value of the radio flux density of the source "between sunspots" that coincides in space with EFR, correlate well.

Particular emphasis should be placed on the AR No. 5629 which produced many flares, including the strongest flare for the whole period of X-ray observations, of importance X20, on August 16, 1989. By analyzing our observational data, we confirmed the conclusion drawn by Li Weibao (1991) that in this AR the process of pressing together of the existing sunspots through EFR was occurring.

The data obtained were used to evaluate the change per day in the absolute emerging magnetic flux in the ARs under investigation. In the most flare-productive regions Nos. $5409,5629,5783$, and 5793 , the daily magnetic flux increase was within the range from 4E2 Mx to 9E2 Mx. Such values are at the upper end of the scale of possible values (Howard, 1991) and can be referred to as "fast variations" (Golovko and Kotrc, 1992).

A correlative analysis of the optical and radio data has shown that the flare-productive active regions were characterized by emerging flux regions evolving with high magnetic flux rates; the EFRs being spatially coincident with conspicuous $\mathrm{cm}$ radio sources "between sunspots". This confirms the result obtained recently by Borovik et al. (1989).

I am grateful to Mr. V. G. Mikhalkovsky for his assistance in preparing the English version of the manuscript.

\section{REFERENCES}

Akhmedov, Sh.B., Bogod, V.M., Gelfreikh, G.B. and Korzhavin, A.N. 1986, in Solar Maximum Analysis, V.N.U. Science Press, p.51

Borovik, V.N., Drake, N.A. and Golovko, A.A. 1989, in Solar Magnetic Fields and Corona. Proceedings of the XIII Consultation Meeting on Solar Physics, v.II, Novosibirsk, "Nauka", Siberian Division, p.162.

Bumba, V. 1986, Bull. Astron. Inst. Chechosl., 37, p.281.

Golovko, A.A., Kotrc P. 1992, Solar Physics (in press).

Hovard,R. 1991, Solar Physics, 131, p.239.

Li Weibao. 1991, Paper presented at Soviet-Chinese Seminar on Solar Phusics. 
Irkutsk, 17-25 May, 1991.

Rust, D.M. 1976, Solar Physics, 47, p.21.

Smolkov, G.Ya., Krissinel, B.B., Treskov, T.A., Potapov, n.n., and Miller, V.G. 1990, Issled. Geomagn. Aeron. Fiz. Solntsa, 91, p.146 (in Russian).

TABLE I Parameters of active regions

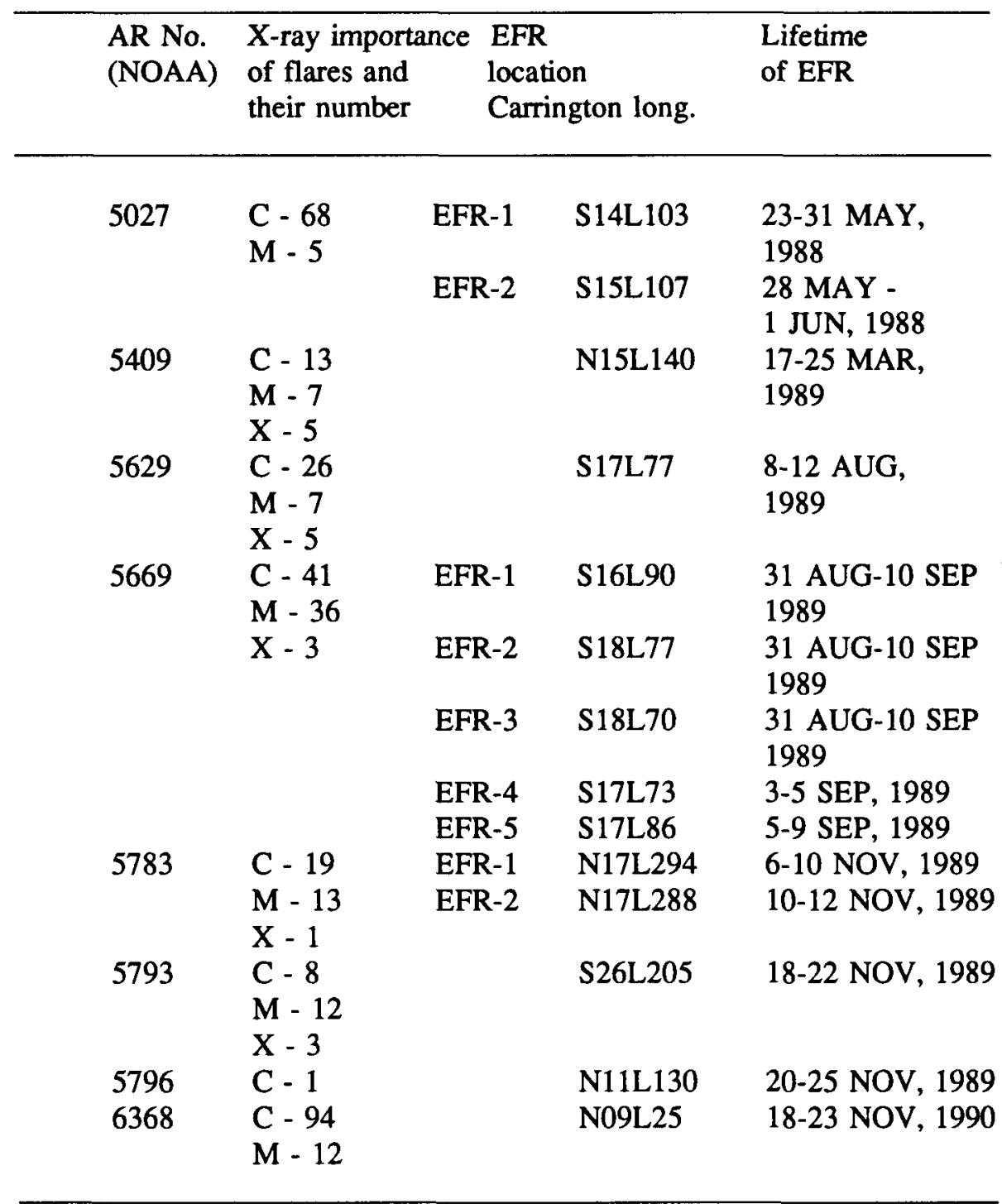

